\title{
Ma langue, mes langues, mélanges
}

\section{Ching Selao}

\begin{abstract}
In this text, Ching Selao writes about her experiences of maternal languages from a perspective both critical and creative. While pointing out the paradoxical way in which languages are represented in Québec, she reveals the bitter sweet pain of feeling on the margins of the French language and of Québécois society.
\end{abstract}

«Je n'ai qu'une langue, ce n'est pas la mienne», a écrit Jacques Derrida dans $L e$ Monolinguisme de l'autre ou La Prothèse d'origine, essai sur la langue française s'ouvrant sur cette proposition antinomique qui souligne que toute langue, $s a$ langue, n'était pas la sienne, mais celle de l'autre. Derrida révélait par là l'origine de ses souffrances, de l'impossibilité d'habiter cette langue alors qu'elle l'habitait, le hantait, du sentiment de ne rester qu'au bord de la langue française, sa langue, celle-là même qu'il n'avait jamais pu appeler «sa langue maternelle» (61): «Or jamais cette langue, la seule que je sois ainsi voué à parler, tant que parler me sera possible, à la vie à la mort, cette seule langue, vois-tu, jamais ce ne sera la mienne. Jamais elle ne le fut en vérité» (14). Au-delà de sa douleur en tant que «Juif indigène» d'Algérie à qui on avait imposé une langue étrangère, Derrida insistait sur la non-propriété d'une langue, car si la seule langue qu'il parlait n'était pas la sienne, elle n'était pas non plus celle de l'oppresseur qui ne la possédait pas naturellement, mais qui se l'était appropriée «au cours d'un procès non naturel de constructions politico-phantasmatiques» (45). Or, si Derrida, Francomaghrébin né à El-Biar mais devenu philosophe français internationalement reconnu, qui nous a malheureusement quitté, a pu écrire qu'il n'y a pas de propriété de la langue, m'est-il possible, moi, petite réfugiée devenue naturalisée à qui on a appris la merveilleuse langue française, de dire, ici, au Québec, que les Québécois n'ont pas de langue, que ce pour quoi ils n'ont cessé - et ne cessent encore - de se battre ne leur appartient pas, que ce qu'il croit être leur langue n'est rien de plus qu'une appropriation fantasmatique leur permettant de fantasmer sur une identité non moins fantomatique? Et de quel droit dirais-je ceci alors que tous rêvent d'une langue maternelle? Derrida lui-même avait beau insister sur la non-propriété d'une langue, lui aussi s'était approprié sa langue, la française qu'il n'avait pu appeler la maternelle et qui demeurait le lieu de son fantasme.

Pour qu'elle soit langue, la langue française doit peut-être demeurer un fantôme, un spectre qui nous habite et nous hante, un fantasme faisant du parler une expérience corporelle: «L'expérience poétique n'est pas “je parle la langue", mais "la langue me parle", la langue parle à travers moi» (Lê 2005, 9192). La langue me parle, la langue me touche, la langue fait corps avec moi, elle me pénètre et s'échappe de ma bouche. Mais la langue avec laquelle j'entretiens 


\section{$26 \cdot$ Tessera}

ce rapport charnel est-elle ma langue maternelle? Quelle est, au juste, ma langue maternelle? Le dialecte de mes parents? La langue de celle qui a joué avec moi à la mère? Ou encore celle du pays où je suis née, mais où je n'ai vécu que quelques années, et qui n'est d'ailleurs pas le pays de mes parents? N'ai-je pas été bercée par plusieurs langues maternelles, langues que je me suis toutefois efforcée de ne pas parler une fois les pieds sur le sol québécois? Ma langue est celle-là même que je me suis appropriée comme une prothèse pour remplacer celles qui n'ont jamais été miennes. Plus jeune, il m'arrivait souvent, pour ne pas dire toujours, d'employer des mots de trois ou quatre langues dans une seule phrase, croyant que je parlais $m a$ langue. Peut-être est-ce celle-là ma langue, un mélange de mes langues: «Je n'ai qu'une langue, ce n'est pas une langue.» Aujourd'hui, ces langues, je les parle à peine et seulement en milieu familial et seulement avec ceux qui ne parlent pas, ou à peine, les langues d'ici, ces langues qui contaminent «mes» langues maternelles.

$\mathrm{J}$ 'ai laissé le territoire des langues d'origine pour m'installer dans une autre langue, dans la langue de l'autre, une langue qui ne m'est pourtant pas (ou plus) étrangère. Cette idée, d'autres, comme Derrida, l'ont exprimée avant moi et mieux que moi. Mais quitter des langues sans que celles-ci ne nous quittent, $c$ 'est précisément porter en soi le deuil impossible des langues maternelles qui, refoulées, enfouies, resurgissent au moment où l'on s'y attend le moins. Julia Kristeva a beaucoup écrit sur la langue maternelle qui ne meurt jamais, malgré le besoin, malgré le désir de s'en débarrasser. On peut l'étouffer, la cacher comme un enfant handicapé (1991, 26-27), la porter comme une honte à peine cachée puisque parfois le physique trahit déjà cette honte, la langue originaire, nous dit Kristeva, ne peut s'anéantir, se cadavériser (1992, 27-28). Et peut-être meurt-on le plus souvent de solitude dans notre désir de la tuer. Le propos de Kristeva est à la fois tragique et merveilleux, troublant et rassurant, il est, en tout cas pour moi, vrai. En effet, lorsque la situation l'exige, il m'arrive souvent de chercher mes mots maternels, alors que lorsque je me crois réellement installée dans la langue française, dans le territoire sûr et dominant de l'illustration de cette magnifique langue, un mot, une expression s'immisce dans mon parler ou mon écriture pour me rappeler que j'ai beau vouloir enterrer «mes» langues au fond de moi, jamais je ne pourrai en faire le deuil. Jamais elles ne me quitteront. J'ai quitté mon pays natal, qui n'est pas mon pays d'origine, pas celui, donc, où mes parents sont nés, dans les vomissements d'une vingtaine d'heures de vol; $j$ 'ai vomi ce pays dont je garde à peine quelques souvenirs et dont je pourrai dire que j'ai fait le deuil, malgré l'autre pays qui me hante toujours, mais je n'ai jamais quitté le territoire de mes langues maternelles. Ou plutôt, ce sont elles qui ne m'ont jamais quittée. Je suis partie de mon pays natal, le laissant derrière moi, tandis que mes langues maternelles m'ont suivie pour que je n'oublie pas que je venais d'ailleurs. On ne m'a pas non plus laissé la chance d'oublier que je portais en moi d'autres langues, d'autres couleurs, d'autres racines. On n'oublie pas ici, on se souvient.

Et moi aussi je me souviens. Je me souviens que pour faire oublier mes origines, il m'a fallu oublier ces langues, oublier qu'il était possible de parler d'autres langues que celle d'ici, oublier qu'il était possible de parler autrement la 
langue d'ici. Je me souviens de ces années où j'étais mal dans ma peau jaune, où je cachais ma honte derrière un air prétentieux devant les «miens» et sous un accent si «pur» qu'on aurait pu me prendre pour une «vraie» Québécoise. Et on me prenait pour une «vraie» Québécoise, tant qu'on ne me voyait pas.

Alors que l'histoire du Québec a toujours tourné et tourne encore autour des langues, ma petite histoire à moi a longtemps tourné autour des accents. Derrida a également parlé de son obsession de l'accent «pur» dans Le Monolinguisme de l'autre. À l'instar de Derrida qui, dans cet essai, a osé avouer l'inavouable (78), longtemps, je n'ai supporté que l'accent «pur», qui n'est cependant pas le même accent dont il parlait puisque, dans mon cas, il s'agissait d'une "pureté» de l'accent d'ici, du Québec, de ce lieu où j'habite et à partir duquel j'écris, alors que pour Derrida, c'était la pureté de l'accent de là-bas, de la France, plutôt contesté ici. Mais comme pour Derrida, mon désir d'un accent «pur» trahissait un complexe, non celui du colonisé mais celui de l'immigrée. Toujours, j'ai eu envie de l'accent québécois, envie d'un corps à corps non seulement avec la langue, comme l'a magnifiquement décrit Abdelkebir Khatibi dans Amour bilingue, mais avec l'accent "pur» pénétrant en moi afin qu'à travers lui, mon corps ne soit plus perçu comme un objet d'impureté. Je me suis, autant que possible, frottée à cet accent. Mais ai-je vraiment eu le choix dans le milieu blanc exclusivement francophone dans lequel $\mathrm{j}$ 'ai grandi? Avec quels autres accents, quelles autres langues aurais-je pu me coller pour me consoler de ma solitude? Une solitude que même la complicité de mes frères et sœurs, la présence d'amis et une «popularité» à l'école n'arrivaient à atténuer. Si pour Linda Lê, cette écrivaine que $\mathrm{j}$ 'aime et qui manie la langue française comme $\mathrm{j}$ 'aimerais le faire, "écrire dans une langue qui n'est pas la sienne, c'est faire l'amour avec un cadavre» $(1999,332)$, pour moi, parler dans la langue qui n'est pas la mienne a longtemps signifié embrasser le fantôme de l'identité qui m'échappait. Certainement, cette envie a fait que j'ai joui de l'emploi du français, un plaisir qui m'a néanmoins coûté cher, puisque ce plaisir ne pouvait être vécu qu'à la condition d'une mise à mort d'une autre langue, d'une autre identité, d'un autre plaisir. Car mon corps à corps avec cet accent, et par conséquent avec cette langue, me condamnait à une expérience douloureuse, celle du deuil inconsolable mais inévitable de mes origines. Deuil d'un plaisir à peine frôlé, deuil d'une jouissance non connue. Perte de ce qui n'a même pas été à moi.

- Tu parles vraiment bien français!

- Merci, vous aussi.

$-? . .$.

- Vous aussi, vous parlez bien français.

- Oui mais, moi, c'est ma langue.

- Ah oui, vous êtes né et vous parliez déjà français? Vous n'aviez pas à l'apprendre? 


\section{$28 \cdot$ Tessera}

Pour plusieurs, cette remarque se présente comme un compliment et ceux à qui elle s'adresse le prennent souvent comme tel. Souvent, mais pas toujours. Que sous-entend ce type de commentaires? D'abord que moi, petite Asiatique aux yeux en amande et au cœur québécois (c'est ce que disait une affiche dans le métro suite au «dérapage» de Jacques Parizeau), peux étonnamment bien parler le français (est-ce à supposer que le cœur québécois se greffe plus aisément que sa langue?); ensuite que moi, petite Asiatique aux yeux en amande et au cœur québécois, malgré cette greffe, ne trompe personne. «Tu parles vraiment bien français!» et «Tu parles vraiment comme nous!», ce genre d'affirmations que d'aucuns qualifieraient de flatteur me flatte parfois dans le mauvais sens des poils. Car «Tu parles vraiment comme nous!», c'est aussi, et peut-être surtout, me rappeler que je ne fais pas partie de ce «nous» puisque «Tu parles vraiment comme nous!» veut également dire «Tu parles comme nous pour quelqu'un qui n'est pas nous». Autrement, pourquoi aurait-on besoin de me dire que je parle comme les Québécois si j'étais une «vraie» Québécoise? Ces mêmes personnes prennent-elles le temps de dire à tous les Québécois qu'ils parlent comme elles?...

La culture et l'identité nationales, on le sait, se définissent au Québec par la langue. Mais est-ce vraiment la langue française qui, dans ce pays de neige, définit l'identité? À lire Régine Robin et Flora Balzano, on peut en douter. Dans Soigne ta chute, la narratrice de Balzano n'exprime-t-elle pas sa difficulté d'être Québécoise justement à cause de la langue française, $s a$ langue mais parlée autrement, avec un accent différent, le «vrai», le «bon» selon les Français? «On ne devient pas Québécoise. On ne devient rien.» (31), écrit cette "Québécoise pure acrylaine» (56). Cette boutade, renvoyant à la célèbre phrase de Simone de Beauvoir, avait en quelque sorte été immortalisée par Régine Robin dans La Québé coite, dont la narratrice, à défaut d'être Québécoise, devenait québécoite, perdant la parole à force de se justifier: «ON NE DEVIENDRAIT JAMAIS VRAIMENT QUÉBÉCOIS./ De l'autre côté de la barrière linguistique?/ Allons bon. Elle serait venue de Paris/ pire encore/ maudite Française./ Un imaginaire yiddishophone? Quel drôle de mot!» (37). Il est étrange comme on devient facilement «objet»: «On ne naît pas femme, on le devient» (De Beauvoir 285), "On ne naît pas Nègre, on le devient» (Laferrière 163) et «On ne naît pas écrivain-migrant, on le devient» (Ollivier 2001, p. 69), alors qu'on ne devient pas, ou difficilement, «sujet» (Robin et Balzano). Même un sujet anciennement objet. Si les Québécois n'aiment pas toujours l'accent immigrant, comme celui de mes sœurs aînées par exemple (qui, du reste, ont de la «chance» de travailler dans une usine de textile où leur accent, mêlé aux autres accents, est à peine perceptible), ils supportent peut-être encore moins l'accent français, comme celui des personnages de Robin et de Balzano par exemple (oui, je sais, les Français peuvent être bien pires envers les Québécois). Lorsqu'on me dit «Tu parles vraiment comme nous», on ne veut pas $m$ 'indiquer que je parle la même langue que les Québécois francophones, en l'occurrence le français, mais que je parle le français avec l'accent d'ici. Un autre immigrant, habitant ici depuis autant d'années que moi et parlant la langue française mieux que moi, mais avec un accent qui n'est pas celui d'ici, ne s'est sans doute pas fait dire aussi souvent à quel point il parle bien français. Lors d'un colloque à 
Québec, dans la capitale, un professeur d'université à la retraite, que je ne connaissais que de réputation, m'a même pris dans ses bras après la présentation d'une communication parce que je parlais comme une «vraie» Québécoise...

Mais pour savoir que je parle avec l'accent d'ici ou, comme disent plusieurs, «sans accent», encore faut-il qu'on m'adresse la parole en français. Car il y a la couleur de la peau, de ma peau jaune, idée que ma nièce de 5 ans refuse puisque la pelure d'une banane est jaune, pas ma peau. De toutes mes nièces et de tous mes neveux, il n'y a que celle-ci pour me répondre cela et pour ajouter que ses amies qu'on dit avoir la peau noire ne sont pas noires, mais brunes. La petite croit vraiment que je ne connais pas mes couleurs. Et pourtant, la couleur reste collée à la peau, même dans ce pays de «Nègres blancs d'Amérique», «les seuls nègres aux belles certitudes blanches» (64), comme l'a si bien écrit Jacques Brault. Et ma couleur, comme celle d'autres, fait en sorte qu'il est fréquent que les vendeurs/vendeuses et les caissiers/caissières $m$ 'abordent en anglais, la langue de l'Autre. Ce qui étonne, dans ce scénario très réaliste et réel, c'est que ceux qui font cela ne sont pas que des gens bilingues ou anglophones, mais souvent des francophones qui cherchent parfois maladroitement leurs mots. J'ai beau dire «Bonjour!» plutôt que «Hi!», rien n'y fait, on associe d'emblée mon apparence d'immigrante à la langue anglaise. Émile Ollivier a déjà parlé de son agacement face aux vendeurs/vendeuses - le plus souvent francophones - des magasins du centre-ville qui l'abordaient en anglais (1990, 48), lui qui se disait Québécois le jour et Haïtien la nuit et qui parlait et écrivait merveilleusement dans la langue de Molière. Au centre-ville ou dans le quartier où j'habite, la chose me paraît aujourd'hui moins étonnante - je ne dis pas «normale» - puisqu'il faut le dire: de nombreux immigrants, même lorsqu'ils parlent également français, aiment parler anglais dans la vie de tous les jours. Et moi-même, je l'admets, n'en déplaise à ceux qui croient que je devrais ne parler qu'une seule langue, celle-là même que l'on me rappelle sans cesse ne pas être la mienne, il m'arrive, dans mon intimité et avec certaines personnes, d'employer cette langue. Ce qui devient par ailleurs un problème, ce qui est presque une insulte, c'est lorsque les gens de la banlieue du nord s'obstinent à me parler en anglais alors que, de toute évidence, ils ne maîtrisent pas cette langue. N'est-ce pas leur façon à eux de me dire que leur anglais, aussi médiocre soitil, sera toujours supérieur à mon français? Bien sûr, pour quelques-uns, ce n'est que l'occasion de pratiquer cette langue, mais je ne parle pas d'eux, je parle des autres, de ceux pour qui «ma» langue française ne sera jamais mienne.

On regrettera ensuite que les immigrants «choisissent» l'anglais. Car ce genre de situation n'est pas exclusif à la banlieue. Même si en ville, je ne m'étonne plus qu'on me parle en anglais, certaines situations sont tout de même surprenantes. Dans un des restaurants près de chez moi, par exemple, les serveuses francophones me parlent en anglais lorsque je suis avec une personne appartenant à une des minorités visibles (même si j'ai commandé mon plat en français), alors qu'elles s'adressent à moi en français si je suis avec quelqu'un d'apparence de «souche». Il y a environ un an, une personne qui $m$ 'est très chère me racontait un incident qui abonde dans ce sens et qui illustre au mieux mon 
propos. Cette personne, je dois le préciser, trouve que j'analyse un peu trop ce sujet, que ces situations ne lui sont que rarement arrivées. En fait, elle les a vécues assez fréquemment, seulement elle a une confiance en elle qui fait qu'elle accorde très peu d'importance au regard et aux commentaires d'autrui. Donc, cette personne, un jour, s'est rendue chez un médecin spécialiste de Montréal pour accompagner sa mère. En entrant, malgré son «Bonjour!», la secrétaire l'accueille en anglais ce qui, du coup, ne la surprend pas puisque l'anglais est une langue qu'elle parle aussi couramment que le français. Toutefois, en écoutant les explications sur les procédures, elle se rend compte que la secrétaire cherche ses mots, si bien qu'elle lui pose les questions en français. Mais la secrétaire s'obstine, s'entête jusqu'au moment où, dans sa quête de mots, elle ne trouve pas le terme en question et se résigne à parler français à cette personne immigrée. Celle-ci, dont le corps ne ment pas, entre finalement dans le bureau du médecin, toujours avec sa mère, et dit d'emblée «Bonjour!». Ce «Bonjour!» surprend au plus haut point Dr. Francophone:

- Ah! vous parlez français?

- Oui...

- Ah... je croyais que tous les Chinois parlaient anglais!

- Ah... je croyais que tous les Chinois parlaient chinois!....

- ...

En écoutant le récit de cet incident, je jalousais intérieurement cette réplique qui ne venait pas de moi, ironique et pourtant d'une évidence implacable, une réplique à laquelle je n'avais jamais pensé même si ce type de remarque ne m'était pas étranger.

Les francophones ne sont évidemment pas les seuls à associer les immigrants à la langue anglaise, les anglophones aiment davantage se complaire dans cette idée, mais à quoi bon m'étendre là-dessus puisque ce texte, écrit en français, n'intéressera sans doute aucun d'entre eux? Quant aux immigrants, il y en a plusieurs pour qui l'apprentissage du français est une obligation, tandis que l'apprentissage de l'anglais va de soi. Parmi ces immigrants, il y a les gens de ma «race», les «miens», pour qui l'intrusion de mon accent d'ici dans mes langues maternelles traduit une trahison, voire une désertion. Ceux-là, que je les connaisse ou non, se demandent souvent, certains l'expriment clairement, alors que d'autres sont transparents à cause de leur sourire condescendant: comment peutelle si mal parler $s a$ langue? C'est sous ce reproche que les plus vieux, mais surtout les plus jeunes - ceux qui ont perdu la fluidité dans la langue maternelle mais qui n'ont jamais eu la chance qu'on leur dise: «Mais tu parles vraiment comme nous!» - me révèlent leur jalousie, leur amertume de ne pouvoir passer pour une banane (jaune de l'extérieur et blanc à l'intérieur). Mais la banane ne trompe personne, pas même ceux qui voudraient y croire. ll y en aura toujours pour froncer les sourcils lorsque je parle ou lever le sourcil droit, à l'affût d'une erreur qui leur donnerait la satisfaction de me reprendre. Il y en aura toujours pour me rappeler que je ne suis pas d'ici et qu' on ne change pas de peau comme on change de langue. 
En prenant le métro le 14 février 2005 pour me rendre à l'Université de Montréal où j'allais parler d'Aimé Césaire à des étudiants, ce grand poète et dramaturge de la dénonciation des injustices, je me répétais la nécessité de leur préciser que la virulence de ses textes devait être placée dans le contexte des années 50, dans la période des mouvements de décolonisation. À cette pensée, à la pensée que Cahier ou Discours puissent de nos jours paraître excessivement violents, en particulier dans le contexte québécois même si Césaire en a inspiré plus d'un ici, un fou s'est mis à me crier après: "Tu pues sale immigrée! Retourne chez vous! Ostie de niaiseuse, je te souhaite de te faire violer!» En ce jour de la SaintValentin, à la station Snowdon, ce fou qui avait l'air tout à fait normal et que mon regard avait machinalement croisé en entrant dans le train sans penser qu'il déchaînerait ces violences verbales, ce fou, donc, m'a brutalement rappelé qu'on ne change effectivement pas de peau comme on change de langue. Je voulais être invisible, ce jour-là, moi, la minorité un peu trop visible qui continuais à penser à Césaire, à ce qu'il avait écrit et à tout ce qu'il restait encore à écrire. C'est un fou, me disais-je, pour me rassurer. C'est un fou, me répétait tout le monde, par la suite, pour me rassurer. Et j'étais rassurée, car c'est rassurant de penser que seuls les fous disent et pensent ceci.

Des journées comme celles-là me font prendre conscience que «je suis partie mais ne suis pas arrivée» (Chen 35 ). Contrairement à Ying Chen, cependant, qui réalise «non sans bonheur» (35) qu'elle n'arrivera peut-être jamais, moi, dont l'avion est atterri depuis près de 25 ans, $\mathrm{j}$ 'aimerais bien arriver un jour. Si les théories et la littérature aiment nourrir l'idée que l'étranger ne vient de nulle part et ne va nulle part, entretiennent les notions de l'entre-deux, des errances, du no man's land et du no man's langue, il me semble que dans la réalité, on aspire à autre chose. J'aimerais bien savoir qui, dans un camp de réfugiés, rêve au no man's land ou à l'entre-lieu. J'aimerais bien connaître un seul immigrant qui, à l'éternelle question «D'où venez-vous donc?», a pu répondre: «Mais de Nulle Part!...» Car dans le quotidien, on vous rappelle, souvent insidieusement, parfois violemment, que vous venez de quelque part, d'ailleurs surtout, que vous n'êtes pas d'ici, et que vous pouvez toujours rêvez d'écrire dans un no man's langue comme Gaston Miron ou Marco Micone, avec le risque d'aphasie et le danger du silence (Gauvin 1997, 2000), en attendant la venue à l'écriture, parlez donc dans notre langue et avec notre accent.

Alors que l'injonction se fait sentir un peu partout, l'engouement superficiel pour les langues exotiques est paradoxalement partout présent. Un peu comme les théories, la publicité me fait également croire qu'il est bien de mettre en valeur mes origines et ma différence. Lorsque je pense à ma honte des années antérieures et à celle plus récente de ce 14 février et qu'en revanche, je vois, depuis quelques années, déferler devant moi des images qui me font croire que

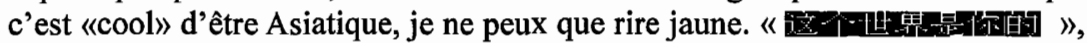
m'annonçait Dex un jour, dont le message était imprimé en gros caractères sur un autobus de Montréal, dans cette langue que je ne lis pas et que les gens à qui $s$ 'adresse le message ne lisent pas plus que moi. «Le monde t'appartient». Je me demande dans quelle langue Dex s'adressait à sa clientèle lorsque le monde autour 
de moi était si blanc, si unilingue monolingue, si peu ouvert à l'altérité. Lorsque cette langue qu'il utilise comme publicité n'était pas encore tatouée sur les corps de plusieurs, fiers de montrer qu'ils s'ouvrent à la diversité culturelle, même si certains n'ont jamais mis les pieds dans le Chinatown de peur de se faire servir des chats ou des chiens. Dernièrement, c'est Nike qui a fait le grand éloge des différences, dans une pub qui montre un joueur de basket-ball, à la peau noire bien sûr, et un narrateur en voix off parler cette langue maternelle que j'ai rejetée pendant toute mon enfance et mon adolescence et que je n'avais jamais entendue à la télévision ni à la radio. Tampax aussi, récemment, s'est mis de la partie. Dans la rue, depuis quelques années, $j$ 'entends des gens, des hommes toujours, m'interpeller et me dire bonjour dans cette langue devenue à la mode. Même M. Sympatico qui fait du porte-à-porte pour s'assurer que tous les services de la compagnie qu'il représente sont toujours «Bell et bien simple[s]» m'accueille avec un bonjour dans cette langue. La chose se produit quelquefois entre les murs des universités. Cet homme, par exemple, à la peau et aux cheveux blancs, rencontré dans l'ascenseur en descendant des départements d'Études françaises et de Littérature comparée, qui s'est mis à me parler dans cette langue. Celui-là, que j'appellerai M. Professeur de l'Ascenseur, je lui pardonne, car bien que je me disais qu'avec tous les Asiatiques qui prenaient cet ascenseur, il ne pouvait quand même pas tous les aborder dans cette langue, je voyais bien que «ma» langue lui appartenait davantage qu'à moi. Il n'y a pas à dire, le monde m'appartient, mais «ma» langue, si elle fut mienne un jour, ne m'appartient plus.

Je ne suis évidemment pas contre l'apprentissage et la circulation des langues. Et puisque j'ai débuté ce texte avec Derrida, je sais bien qu'il n'y a pas de propriété des langues. D'ailleurs, aurais-je voulu croire à cette illusion, à ce mythe, que M. Professeur de l'Ascenseur m'aurait détrompée. Enfant, j'étais fascinée lorsque, assise à une table d'un des restaurants du Chinatown, j'entendais des Occidentaux parler cette langue que je m'efforçais de désapprendre. Je ne comprenais pas pourquoi un Québécois ou un Canadien prenait le temps d'apprendre une langue aussi compliquée, alors qu'elle ne lui permettait que de parler aux serveurs des restaurants du Chinatown. Et même pas à tous les serveurs. Cette fascination, je la ressens de moins en moins: d'une part, parce que ces hommes que j'entendais petite sont de moins en moins rares, d'autre part, parce qu'il me semble que nous assistons de plus en plus non pas à un apprentissage d'une langue que quelqu'un trouverait belle, mais à une exotisation, voire à une commercialisation de cette langue.

Mon rapport aux langues, à mes langues, a beaucoup à voir avec ma relation avec mon père, de sorte qu'il m'est impossible de ne pas l'évoquer. Peut-être s'étonnera-t-on de la nécessité de parler de mon père dans un article sur les langues maternelles, mais tout comme pour Linda Lê, ma langue maternelle, c'est-à-dire celle que $\mathrm{j}$ 'associe au monde de mon enfance, est liée au père. Le mien ne parle qu'un dialecte et bien que ce dialecte soit également celui de ma 
mère, celle-ci, à la différence de mon père, parle aussi une autre langue, une «vraie», si vraie que le mot qui la désigne signifie littéralement «langue nationale». Et de ma mère, je n'ai que peu de chose à dire, si ce n'est qu'elle est la grand-mère, pas toujours tendre mais toujours aimante, que je n'ai jamais eue. Mon rejet de la langue maternelle vient du rejet de mon père, père arrivé trop vieux et n'ayant jamais réussi à apprendre la langue d'ici, père qui - je tiens à le souligner - n'a rien du «petit Chinois» qui caractérise tous les Asiatiques dans l'imaginaire occidental et que $\mathrm{j}$ 'incarne moi-même. Mon père et moi avons toujours eu de la difficulté à nous comprendre, peu importe la langue: mon père et moi, nous ne parlons pas la même langue. Les sociologues ou psychologues de l'immigration diront que c'est très courant, que c'est un problème de génération, un conflit interculturel, mais je sais que ce n'est pas ça, que ça n'a jamais été que ça, que c'est comme Marguerite Duras et sa mère. Sans L'Amant. Sans la Littérature. Dans mon enfance, le français a sûrement été pour moi ce que le vietnamien avait été pour Duras, elle, la petite blanche à la peau douce de l'eau de la pluie qui parlait comme les indigènes: un moyen de défier l'autorité parentale. Mais Duras, grâce aux livres, n'a jamais rompu avec sa langue maternelle et, à l'inverse de sa situation, mes langues maternelles n'avaient (et n'ont toujours) aucun pouvoir dans ce pays.

Mon père ne lira pas ces pages, même si je décidais un jour de les faire traduire dans sa langue. Il ne lira aucune des pages encore à écrire sur lui. Mes parents ne lisent pas plus leur langue qu'ils ne parlent «ma» langue, ce sont des illettrés, des analphabètes avec un grand A. C'est ça aussi mon héritage, l'origine de ma blessure. Pourtant, sans le savoir, mon père me liait, dès ma naissance, à la littérature avec ce prénom qu'il m'a donné, nom des plus shakespeariens, venant de lui, l'analphabète avec un grand $A$, celui qui ne lit pas plus les langues d'ici que celles d'ailleurs: «Pure comme neige», comme l'Ophélie d'Hamlet, et à l'instar de la noyée, "aussi chaste que la glace, aussi pure que la neige, [je] n'échappera[i]s pas à la calomnie» (Shakespeare 109). Ce beau nom qui est le mien, mutilé dans sa translittération, a été ma première violence exilique, le premier effacement d'une identité qui jamais ne pourra être «pure». Etre ou n'être pas pure dans ce pays de neige. C'est la question. Pure comme neige à l'image d'Ophélie ou Neige noire à l'image du lieu de mort, lieu du meurtre-suicide de la Sylvie d'Hubert Aquin? Une chose est sûre, dès la naissance, ce prénom aux résonances désormais exotiques annonçait deux réalités: le départ vers un pays de neige où précisément le mot neige de mon nom allait disparaître, se fondre dans la neige d'ici, et la mise à mort identitaire, car le blanc de la neige est aussi couleur de deuil dans «ma» culture. À un seul mot près et c'est la mort, la mise à mo(r)t de l'identité. Le nom de mon père, le don de mon père dans ma langue maternelle préfigurait, sous le soleil et la chaleur de mon pays natal, un deuil d'une identité à venir et pourtant déjà avortée. Le nom que je porte, celui que j'ai porté dès mon arrivée dans ce pays, $m$ 'inscrivait déjà dans un exil de mes langues maternelles. En exil dans ces langues avant même d'en apprendre une autre. En exil dans ce pays de neige noire, en exil dans l'écriture à venir: l'encre noire sur la page blanche neige. En choisissant ce prénom, mon père, ce roi Lear sans 
pouvoir qui n'a été abandonné par aucune de ses filles, incapables de délier la corde qui les lia à lui, ne connaissait pas l'existence du grand dramaturge anglais. Il n'avait certainement pas pensé qu'un jour, sa cadette, née l'année où les communistes ont envahi le pays natal de ses enfants qui n'est pas le sien, parlerait mieux les langues de Shakespeare et de Racine que ses langues maternelles.

Depuis le jour où la toute petite réfugiée que j'étais, arrivée et recueillie presque nue en pleine tempête (Ah! comme la neige a neigé ce jour-là!) et cet instant où j'écris ces pages, beaucoup de choses ont changé. J'ai, depuis, appris le savoir-vivre d'une société civilisée et je ne suis plus celle que l'on peut reléguer dans l'exotisme «pur» (et «dur»), puisque j'incarne à présent l'exotisme familier, l'inquiétante étrangère à la fois si différente et si familière. En tant que personne naturalisée et évoluée, je sais qu'il n'est pas facile de parler des langues, de ses contradictions, en particulier au Québec, d'écrire sur une douleur autre que celle magnifiquement exprimée dans la poésie de Miron ou dans celle, plus discrète mais tout aussi brillante, de Brault. Il n'est pas facile de «speak» lorsqu'on sait que certains ont signifié à Marco Micone, par des accusations de plagiat, «Thou shall not speak» (ce qui ne l'a d'ailleurs pas intimidé), après sa réécriture du fameux poème «Speak White» de Michèle Lalonde. Micone est pourtant celui qui, contrairement à moi, est arrivé avant la loi 101 et a dû se battre pour apprendre cette langue; il est l'écrivain immigré dont le parcours souligne bien les paradoxes de la défense et de l'illustration du français. Je n'ai certes ni le courage ni la conviction politique de Micone, mais j'ai tout de même voulu écrire un peu sur ce thème qui m'est cher, au risque de voir ces pages sombrer dans «la langue du silence et de l'impuissance» (Micone 85). Je ne prétends à aucune vérité absolue, puisqu'il s'agit d'exprimer mon rapport, tout à fait personnel, à mes langues et mon opinion, tout à fait subjective, d'une certaine représentation des langues dans la société. Ce texte, je l'écris dans «ma» langue qui n'est pas mienne, dans la seule langue qui me relie à celui qui, tout au long de cette expérience, m'a suivie comme une ombre paternelle, celui qui n'avait toutefois rien en commun avec mon père, si ce n'est qu'ils sont tous deux nés au mois de juillet, mon père ayant vu le jour trois ans avant Derrida. Ce texte, donc, n'a pu qu' effleurer le sujet des langues maternelles, car s'il relate mes expériences, il est d'abord et avant tout une expérience, au sens étymologique du terme, c'est-à-dire d' «experi»: «tourner autour, faire erreur» (CalleGruber 233). Écrire dans «ma» langue qui n'est pas la mienne est l'expérience d'une erreur possible, du détour vers d'autres langues, du retour aux langues maternelles, l'expérience, en somme, du tourner autour de l'infini sujet qu'est, au Québec, la question des langues. 


\section{Bibliographie}

Aquin, Hubert. Neige noire. Montréal: Leméac, 1994 [1974].

Balzano, Flora. Soigne ta chute. Outremont: Lanctôt, 2001 [1991].

Brault, Jacques. Poèmes. Montréal: Noroît, 2000.

Calle-Gruber, Mireille. Assia Djebar ou la résistance de l'écriture. Regards d'un écrivain d'Algérie. Paris: Maisonneuve et Larose, 2001.

Chen, Ying. Quatre mille marches, Montréal: Boréal, 2004.

De Beauvoir, Simone. Le Deuxième sexe. Tome 1. Paris: Gallimard, 1949.

Derrida, Jacques. Le Monolinguisme de l'autre. Paris: Galilee, 1996.

Gauvin, Lise. «Une oeuvre d'intranquillité». In Les Adieux du Québec à Gaston Miron. Simone Bussières (dir.). Montréal: Guérin, 1997. 97-99.

Langagement. L'écrivain et la langue au Québec. Montréal: Boréal, 2000.

Khatibi, Abdelkebir. Amour bilingue. Montpellier: Fata Morgana, 1983.

Kristeva, Julia. Étrangers à nous-mêmes. Paris: Gallimard, «Folio/essais», 1991 [1988]. «En deuil d'une langue?». Deuils. Vivre, c'est perdre. Nicole Czechowski et

Claudie Danziger (dir.). Paris: Autrement, 1992. 27-36.

Laferrière, Dany. Comment faire l'amour avec un nègre sans se fatiguer. Montréal: Typo, 2002 [1985].

Lề, Linda. Tu écriras sur le bonheur. Paris: Presses Universitaires de France, 1999. Le Complexe de Caliban, Paris: Christian Bourgois, 2005.

Micone, Marco. «Speak What». Jeu: Cahiers de théâtre 50 (1989): 83-85.

Ollivier, Émile. «Québécois de toutes souches, bonjour!». Vice Versa 28 (1990): 47-48. Repérages. Montréal: Leméac, 2001.

Robin, Régine. La Québécoite. Montréal: Typo, 1993 [1983].

Shakespeare, William. Hamlet. Le Roi Lear. Paris: Gallimard, «Folio/Classique», 1978.

Vallières, Pierre. Nègres blancs d'Amérique. Montréal: Typo, 1994 [1968]. 